\title{
Schema Theory in Reading
}

\author{
Shuying An \\ Changchun University of Science \& Technology, Changchun, China
}

\begin{abstract}
The term "schema" was first used in psychology with the meaning of "an active organization of past reactions or experiences". It assumes that written text does not carry meaning by itself. Rather, a text only provides directions for readers as to how they should retrieve or construct meaning from their own previously acquired knowledge. The theory of Schema can be used to help guide students to comprehend a text from the global point of view. Therefore, the roles of Schema theory in comprehension cannot be ignored.
\end{abstract}

Index Terms - schema, schema theory, reading

\section{Definition OF SCHEMA}

Schema theory is an explanation of how readers use prior knowledge to comprehend and learn from text (Rumelhart, 1980). The term "schema" was first used in psychology by Barlett as "an active organization of past reactions or experiences" (1932,p.201), later schema was introduced in reading by Rumelhalt (1980), Carrell (1981) and Hudson (1982) when discussing the important role of background knowledge in reading comprehension. Rumelhart (1980, P.34) define schema as "a data structure for representing the genetic concepts stored in memory ". Anderson and Pearson (1984, p.42) define it as "an abstract knowledge structure". Medin and Russ (1992, p.246) simply put schema as "a general knowledge structure used for understanding".

The fundamental tenet of schema theory assumes that written text does not carry meaning by itself. Rather, a text only provides directions for readers as to how they should retrieve or construct meaning from their own previously acquired knowledge. This previously knowledge is called the readers' background knowledge (prior knowledge), and the previously acquired knowledge structures are called schemata (Barrlett, 1932; Adamsand Collins, 1979; Rumelhart, 1980). The schemata of a reader are organized in a hierarchical manner, with the most general at the top down to the most specific at the bottom. According to schema theory, comprehending a text is an interactive process between the reader's background knowledge and the text. Efficient comprehension requires the ability to relate the textual material to one's own knowledge. As Anderson (1977, p.369) point out, "every act of comprehension involves one's knowledge of the world as well". Reading comprehension operates in two directions, from bottom up to the top and from the top down to the bottom of the hierarchy. Bottom-up processing is activated by specific data from the text, while top-down processing starts with general to confirm these predictions. These two kinds of processing are occurring simultaneously and interactively, which adds to the concept of interaction or comprehension between bottom-up and top-down processes (Carrel and Eiserhold, 1983).

\section{TYPES OF SCHEMATA}

According to the nature of contents, different types of schemata have been suggested: i. formal schemata, relating to the rhetorical structure of the text; ii. Content schemata, relating to the content of a text read; and iii. Cultural schemata, more general aspects of cultural knowledge shared by larger sections of a cultural population, iv. Carrell(1988) had also added linguistic schemata (Urquhart and Weir, 1998, p.71).

i. A formal schema refers to "background knowledge of the formal, rhetorical organizational structures of different types of texts" (Carrel and Eisterhold, 1983, p.79).In other words, formal schema refers to the knowledge of the ways in which different genres are presented, with reference to Richards et al. (2000, p.405), they point out that schema or macro- structure refers to file underlying structure which accounts for the organization of a text or discourse. Different kinds of texts and discourse (e.g. stories, description, letters, reports, poems) are distinguished by the ways in which the topic, propositions, and other information are linked together to form a unit. This underlying structure is known as formal schemata. For example, the schema underlying many stories is: story=setting (state+state) +episodes (events) +reaction. That is, stories consist of a setting in which the time, place, and characters are identified, followed by episodes leading towards a reaction. Different genres have different structure. Lack of such kind of knowledge also contributes considerably to the problems in reading comprehension.

ii. Content schema refers to "background knowledge of the content area of the text" (Carreli and Eisterhold, 1983, p.80). It contains conceptual knowledge or information about what usually happens within a certain topic, and how these happenings relate to each other to form a coherent whole. It is an open-ended set of typical events and entities for a specific occasion. For example, schema for going to a restaurant would include information about services, menus, ordering dishes, paying the bill (giving a tip), and so on .Content schema are largely culture-specific. Therefore, cultural schema is usually categorized as content schema. 
iii. Richard et al. (2000, p.117) define culture as "the total set of beliefs, attitudes, customs, behavior, social habits, etc., of the members of a particular society". Rivers and Temperly (1978, p.202) call cultural knowledge "socio-cultural meaning" which is "meaning which springs from shared experiences, values and attitudes".

Studies by Johnson (1981), and Carreli (1981), have shown that the implicit cultural knowledge presupposed by a text interacts with the reader's own cultural background knowledge of content to make texts whose content is based on one's culture easier to read and understand than syntactically and rhetorically equivalent text based on a less familial-, more distant culture.

Furthermore, different groups may interpret the same texts differently, as is showed in the study by Steffenson et al (1979). It is important to be sensitive to cultural differences, particularly, of the target culture, and without such cultural awareness there may be no efficient and total comprehension.

iv. Linguistic schema refers to the knowledge about vocabulary and grammar. It plays a basic role in a comprehensive understanding of the text. Eskey (1988, p. 94) claims that "good readers are both decoders and interpreters of texts, their decoding skills becoming more automatic but no less important as their reading skill develops". This is because that "Language is major problem in second language reading, and that even educated guessing at meaning is no substitute for accurate decoding" (Eskey,1988, p.97).In other words, successful comprehension of any text is impossible without effective decoding skills.

\section{SCHEMA ACTIVATION AND INSTANTIATION IN READING}

Schema activation is generally recognized as the process in which some textual stimuli signal the direction or area for the reader to look for and evoke the relevant schema from memory into the present reading task (Li and Cheng, 1997 , p.295-296). One assumption about schema activation is that some words, or groups of words, or the title of a text, are highly suggestive and they can signal a certain schema. Textual stimuli affect a schema in two ways. If a stimulus is highly suggestive of a certain schema, that schema as a whole can be activated. For instance, the mention of a fire brigade may activate a "fire accident" schema. But more often than not, one such stimulus is insufficient for schema activation, it can just remind one of a certain slot which can fit into several schemata. The mention, for example, "acid" can signal a slot for schemata as "acid rain", "food processing", and so on. As more and more stimuli are provided, the possibilities become fewer and fewer and, ideally, the reader may concentrate on the one that is anticipated by the writer. If besides acid, other stimuli such as "litmus paper", "Bunsen burner" and "test tube" are also present, then it is highly probable that a "chemistry lab" schema will be activated.

The other way textual stimuli affect schema is that an activated schema will give schema-specific significance to some of the textual stimuli which otherwise do not have much obvious connection to one another. For example, the relation between "apron" and "chair" seems arbitrary and they may suggest, among other thing, a "kitchen" schema. But with "brush" and "clip", they in fact suggest a "haircut" schema. Once that schema is activated, "apron" and "chair" will be given a schema-related interpretation. Furthermore, other components, whether present in the text or not, which should also be included in that schema will also be recalled, for instance, "hairstyle", "shampoo", and so on.

Schemata instantiation refers to the particularized representation of the general abstract and stereotypical schemata which the reader brings to task. The schemata are abstract in the sense that they contain a slot or place holder for each constituent element in a knowledge structure. They are stereotyped in that they indicate typical relationships among the elements. The reader is involved in a process of constructing a correspondence between the relevant schemata and the givens or knows of a message. The ingredients needed to fill the slots will not always be found in the message itself, but may be reader supplied. As the correspondence is constructed, the reader gains a sense that the message in the input has been and is being comprehended. When the slots of the schemata are filled with enough particular cases, a schema is said to be instantiated. It is known until the schema is instantiated that the representation in the message makes sense and is consistent. In other words, comprehension of a message entails drawing information from both the message and the internal schemata until sets are reconciled as a single schema or message in which the constraints of both the graphic message and the internal schemata are satisfied (Anderson et al., 1977). What is semantically complete will depend upon the amount of completeness needed to reconcile the schemata as defined by the reader. As an example of this process, consider the following three sentences: He picked up his axe. He held it softly for a moment and blew sharp notes through its bell. The crowd listened and cheered him; they loved his saxophone playing. When the first sentence is read, it is interpreted and a schema is produced. However, when followed by the second sentence, the constraints of "axe", "blew", "sharp notes", and "bell" are not reconciled. At this point the reader may either dismiss the initial schema or become confused and continue to attempt reconciliation of the two sentences. Yet, when the third sentence is added, the reader may reconcile the schemata in reinterpreting and intimidating the definition of "axe" as a jazz instrument, namely a "saxophone". Alternatively, the reader can retain the schema from the first sentence and impose a reconciliation which is not correct. It has been indicated that the mental representation of the to-becomprehended sentences is generally more elaborate and detailed than the words in the utterance might appear to entail. Thus, the first image may be so strong that it will not be reinterpreted. This is not a component problem or the result a linguistic ceiling. Only enough slots need to be filled to provide the meaning which leads to reconciliation and recognition. The image may be reconciled and no incoming information allowed filling slots and contradicting the instantiated schema. 
In the 80s, the schema theory of reading, called the integral schema teaching model, began to be introduced into our country according to an essay by Hu Chundong and Wang Cairen (1998) entitled "On English Reading".

The integral schema teaching model emphasizes that reading is the positive intercommunication between reader and writer, and it focuses on the function of background knowledge in reading comprehension. Mr. Liu Longgen (1988) ever said in an essay that rational reading teaching put most of its attention on the linguistic form of the reading material and ignored the function of readers and their background knowledge; hence the development of students' reading ability was restricted. According to Guilian Brown in his The Nature of Comprehension, "the 1990s view of the discourse comprehension' insists that part of the interpretation is formed by inference. Such inferences are drawn on the basis of previous experience of life, or of similar text, or of films or television programs, or on the basis of emphatic

imagination, etc. As each sentence of the text is interpreted by the reader, together with inferences which permit its interpretation in that context, is assimilated into a constantly recreated matrix of ideas. It is sometimes suggested that the main problem for the foreign language learner lays net so much in lack of knowledge of the forms of language as in the lack of background knowledge of the culture and society from the foreign language springs. The schema theory of modem cognitive psychology believes that human's knowledge exists in the long-term memory as the schema forms and develops into a large three- dimensional schema net structure.

According to the integral schema teaching model, the schema refers to the fact that all kinds of knowledge can be grouped into some certain units and the building blocks of cognition. Schemata are hierarchical in that the large schema includes the small one, and the small schema includes the even smaller one. The schema has all kinds of categories, such as affair schema, situation schema, role schema, category schema, story schema and expository schema. (Chen Xiaochun, 1998, p.148-152) For example, "selling" is a scheme of affair schema structure. It includes buyer, seller, currency and the knowledge related to "selling". Those above factors are called variables. When human sensory system receives a message, much related knowledge in the schema net structure will be activated and the schema is used to explain some particular plot, so the variables will be particularized by some specific information. This particularization process is the so- called comprehension process.

According to the integral schema teaching model, there are certain relations between different parts of the schema, which can deepen and further the understanding of the reading material. All schema theories emphasize the non-r Text strategies

It refers to the producer's general decision-making and choice of the text produced in the producing process. While the text-strategic continuity is particular, the writer's choice of the text-strategic continuity is determined by the purpose of communication. In a word, it is the method the author adopts to arrange the layout of the whole text according to the purpose or function of the discourse. The continuity usually has the following styles: I Continuity of time. It is realized by a time chain at the front of the sentence or paragraph, and sometimes non-adverbial time expressions can occur in the time chain, ii. Continuity of place. It is also realized by an adverbial chain, which also includes the continuity of the non-adverbial marker, at the beginning of a sentence or paragraph, iii. Continuity of action .It refers to the continuity composed of the figures, animals, or those who are dealt with as a figure, iv. Continuity of topics. It refers to the continuity composed of the signified of the non-figure or non-animal entity that often occurs in an explanatory or argumental passage, v. Continuity of action. It usually conveys new information. In all, to abstract the text strategy in the discourse reading process not only can set a text strategy schema of a certain style step by step in the reader's mind, but can help the readers decode acroscopically, efficiently and correctly. (Liu Chendan, 1999, p.145-154) Just as Bransgord ever said, to look for meaning of the part from among of the whole, is the essential condition to interpret' the comprehension' from the view of the schema theory. That is to say, if we cannot find the meaning of the part from among the whole, there will be some problems with comprehension. Usually a discourse consists of many affairs according to certain relations and the affairs combine into a topic, which is a large schema. Therefore, whether the reader understands the text or not depends upon if he can find the topic schema. Actually we usually first look for the topic schema when we are reading. To look for the title at first is usually an unconscious behavior, it reflects our reading strategy: first scan the whole, and then try to get the meaning of the parts of from among the whole. Then the explanation of the text will be accurate and reasonable and done more quickly. (Chen Xiaochun, 1998, p.166-167)

The integral schema teaching model seems to be a perfect one to explain the reading process and to be utilized to teaching English reading at a college level, yet it tends to forget that reading occurs under certain circumstances with a definite purpose. As a matter of fact, teachers do train students' reading skills while imparting linguistic knowledge. But these reading skills are trained as separate skills and the students get the wrong idea that they should understand different texts with the same method.

Therefore, though the schema theory guide the students to improve from sensory thinking to imaginative thinking so that students can be active in their process of reading, guessing, confirming the text positively, it pays little attention to vocabulary and basic language points. So reading in this way tends to be a reading process only to acquire information and readers are easy to be satisfied with the general understanding while ignoring language points which need to be mastered.

\section{TeXt Types, TeXts Patterns And TeXt StRategIES}

The text can be classified according to text-external criteria and the text-internal criteria. The text-external criteria are 
related to the communication situation, such as the report, poem, joke, letter, advertisement, note, story, lecture etc, while the text-internal criteria are related to the form and content. A text can be classified as an expository text, a descriptive text, a narrative text, an argumentative text, a persuasive text, an evaluative text, and a procedural text. Kinneavy (1980) grouped text into 3 parts starting from the discourse purpose: the expressive texts with the focus on the author, the persuasive texts with focus on the readers, and the inferential texts with the focus on the topics. And then he further classified texts from the angle of facts: the descriptive texts focusing on the static state, the narrative texts focusing on the dynamic state, and the evaluative texts focusing on whether they have other choices on the current state. (Liu Chendan, 1999, p.125-126) Once readers have got the style of passage, they can make prediction to the passage they are going to read, and then the passive reading can be replaced by the active participating reading. During the reading process, readers can make constant guessing--- exemplifying---guessing--- exemplifying activities and grasp the macroscopic outline all the time, so that they will not trap into the labyrinth of language.

There is, actually, another method to classify texts from the text form and the content structure with the internal criteria. The following are some kinds of the most practical patterns' i. Problem-solution pattern. Take the following passage as an example:

"I was on sentry duty .I saw the enemy approaching. I tried to open fire. The gun's bolt jammed. Staying calm, I applied a drop of oil. That did the trick. I opened fire. I beat the attack." Here,

Situation: I was on sentry duty.

Problem: I saw the enemy approaching.

Solution: I tried to open fire.

Response: I beat off the attack.

ii. Claim-counterclaim pattern. In this pattern, the author first raises a claim or opinion generally accepted or partly accepted, and thus puts forward his own claim or opinion to counter-claim.

For example, "every other critic has said that on food and Cooking is brilliant, a revelation and a unique combination of scientific insight and literacy which sweeps aside all myth and jargon as none have done before. McGee's book is indeed well written, is full of good things and is good to have on the shelves as a continuing source of reference and quotes. But it also has its fair share of mistakes, omissions and misalignments of emphasis." The last comment by the author counter-claims the generally accepted remarks.

iii. Narrative pattern. Nearly all the writings will occur more or less in narrative patterns. The most important example is from Labov (1972)'s narrative structural pattern. He summed up on the base of natural narrative order, six elements: abstraction, orientation, complicating events, evaluation, resolution, and coda. Schiffrin (1981) adopted another pattern based on Labov's. That is, orientation (make the time, space and figure clear), complicating actions (the affairs which compose the story), evaluation (the comment on the story). Resolution (the verdict to the story).

iv. Question-answer pattern. It is similar to the problem solution pattern, but it always sets a question which is distinct and express in a questioning mode. And the development of the text is mainly to search a satisfying answer to the question.

v. General-specific pattern. It can also be called as general-particular pattern, general-example pattern, and preview-detailed pattern. In practical text, one pattern can occur independently, or combined with other patterns to form larger text. McCarthy (1993) argues that the macroscopic structure of this pattern can generally be divided into two categories. The first one is shown as: general statement---detailed statement 1---detailed statement 2---detailed statement 3---detailed statement 4---detailed statement 5----general statements. The second one is shown as: the general statement ---detailed statement ---more detailed statement--- more detailed statement (than the former one )---more detailed statement (than the former one )---.....---general statement. (Liu Chendan, 1999, p.129-130)

These above text patterns are abstract frameworks, but they truly reflect the major features of the English discourse. Readers will surely be efficient in understanding the meaning of a discourse if they are familiar with the basic structural patterns.

Text strategies refers to the producer's general decision-making and choice of the text produced in the producing process. While the text-strategic continuity is particular, the writer's choice of the text-strategic continuity is determined by the purpose of communication. In a word, it is the method the author adopts to arrange the layout of the whole text according to the purpose or function of the discourse. The continuity usually has the following styles: i. Continuity of time. It is realized by a time chain at the front of the sentence or paragraph, and sometimes non-adverbial time expressions can occur in the time chain, ii. Continuity of place. It is also realized by an adverbial chain, which also includes the continuity of the non-adverbial marker, at the beginning of a sentence or paragraph, iii. Continuity of action .It refers to the continuity composed of the figures, animals, or those who are dealt with as a figure, iv. Continuity of topics. It refers to the continuity composed of the signified of the non-figure or non-animal entity that often occurs in an explanatory or argumental passage, v. Continuity of action. It usually conveys new information. In all, to abstract the text strategy in the discourse reading process not only can set a text strategy schema of a certain style step by step in the reader's mind, but can help the readers decode macroscopically, efficiently and correctly. (Liu Chendan, 1999, p.145-154)

In conclusion, the reading schema mainly has three functions as follows: i. Anticipating Function. With the schemata, the readers can guess the type of the text, can have different anticipation towards the topics of different types of texts, 
and especially, the readers can guess the latter context of the text with the help of the former context of the text. ii. Supplementary Function. When readers find certain specific or essential information is insufficient in the reading material. They will activate the corresponding psychological schema and supplement the information related to the material during the reading process, iii. Selective Process. One aspect of this function is that when the schema is activated during the reading process, it constantly selects the most appropriate part to explain the reading material from the schema net structure. Another aspect is that when reading is finished, the schema will help to sort up its own instrument. The three functions relate to each other and work together to understand the text.

Reading is a dominant skill in learning. With the development of cognitive science, especially the cognitive psychology and information processing theories, many applied linguists and psychologists place different weight on reading. They tend to describe reading as a complicated process, which acquires an active involvement rather than a passive one. Some researchers have become concerned about reading process rather than products. In consequence, three models of reading have been proposed to account for the comprehension process, such as bottom-up model, top-down model, and interactive model. It is the interactive model that fundamentally promotes the development of theories in reading, especially schema theory. In the schema-theoretical view reading is an interactive process. The interaction occurs at three levels: interaction between bottom-up and top-down processing, that between lower-level and high-level skills, and between reader's background knowledge and the background knowledge presupposed in the text. Bottom-up processing is activated by specific data from the text. Top-down processing starts with general predictions based on higher level schemata, then searches in the more specific level to confirm these predictions. Bottom-up processing and top-down processing always occur simultaneously and interactively in reading. Readers consciously or unconsciously use the two types of processing interchangeably to construct comprehension. Schema theory guides readers as they make sense of new experiences and also enable them to make predictions about what they might expect to experience in a given context.

\section{REFERENCES}

[1] Adams, M.J. and Collins, A.M. (1979). "A schema-theoretic view of reading" in Fredolle, R.O. (ed.) Discourse Processing.' Multidisciplinary Perspectives. Norwood N. J. Ablex.

[2] Anderson, R.C. et al. (1977). "Frameworks for comprehending discourse". American Educational Research Journal 14(4): 367-381

[3] Anderson, R. C. (1978). "Schema-directed processes in language comprehension "in Lesgold, A.et al(eds) cognitive psychology and instruction. New York: Plenum.

[4] Carrell, P. L.et al.(eds.) Interactive Approaches to second Language Reading. Cambridge: CUP.

[5] Barlett, F.C. (1932). Remembering: A Study in Experimental and Social Psychology. London: New Psychological Linguistics. Shanghai: Shanghai Foreign Education Publication.

[6] Hu Chundong and Wang Cairen. (1998). On English Reading. Guangxi: Guangxi Education Publication.

[7] Huang Guowen. (1987). Essentials of Text Analysis. Hunan: Hunan Education Publication.

[8] Liu Chendan. (1999). Text Linguistics for Teachers. Shanghai: Shanghai Foreign Education Publication.

[9] Liu Longgen. (1998). Cognition Frame Theory and Foreign Language Teaching. Foreign Language: 1, 1-8

Shuying An was born in Shandong, China in 1971. She received her master degree in Foreign linguistics and Applied linguistics from Changchun University of Science \& Technology, China in 2004.

She is currently an associate professor in the School of Foreign Languages, Changchun University of Science \& Technology, Changchun, China. Her research interests include psycholinguistics and Teaching Strategy. 